\title{
Pre-routing Estimation of Shielding for RLC Signal Integrity*
}

\author{
James D. Z. Ma, Arvind Parihar, and Lei He \\ ECE Department, University of Wisconsin, Madison, WI 53706
}

\begin{abstract}
The formula-based $K_{\text {eff }}$ model is a figure of merit for the inductive coupling, and has been used to solve the simultaneous shield insertion and net ordering (SINO) and simultaneous signal and power routing (SPR) problems. In this paper, we first show that the $K_{\text {eff }}$ model has a high fidelity compared to the SPICE-computed noise under an accurate RLC circuit model. We then develop simple yet accurate formulae to estimate numbers of shields needed by optimal SINO solutions under the $K_{\text {eff }}$ model. Extensive experiments show that our pre-routing estimation has errors less than 10\% compared to solutions given by detailed SINO algorithms. These formulae can be used effectively as a pre-routing congestion estimation for layout planning and synthesis.
\end{abstract}

\section{Introduction}

It is becoming increasingly evident that on-chip inductance, especially mutual inductance, should be considered for high-performance interconnect design. Several recent work have studied interconnect optimization considering inductive coupling for multiple coupled nets. Examples include the simultaneous shield insertion and net ordering (SINO) problem [4] and simultaneous signal and power routing (SPR) problem [6].

The $K_{\text {eff }}$ model but not noise voltage is used in $[4,6]$ to represent the inductive coupling. Despite that the $K_{\text {eff }}$ model is far away from accurate, our first contribution in this paper is to show that for optimal SINO solutions, the $K_{\text {eff }}$ model has a high fidelity compared to SPICEcomputed noise under an accurate RLC circuit model. I.e., a SINO solution with a higher coupling under the $K_{\text {eff }}$ model also has a higher noise voltage.

To integrate the SINO problem into the current design flow, it is desired to have an efficient interconnect congestion estimation. Our second contribution of this paper is to develop simple yet accurate formulae to estimate numbers

*This research was partially supported by NSF CAREER Award CCR0093273 and a grant from Intel. Address comments to he@ece.wisc.edu. of shields needed by optimal SINO solutions without running SINO algorithms.

The rest of this paper is organized as follows: Section 2 introduces preliminaries and reviews the related research. Section 3 studies the fidelity of the $K_{\text {eff }}$ model. Section 4 derives formulae for the pre-routing congestion estimation. Section 5 concludes the paper and discusses ongoing work.

\section{Preliminaries and related work}

Similar to [4], we denote a signal net as $s$-wire and define that two nets $s_{1}$ and $s_{2}$ are sensitive to each other if a switching signal on $s_{1}$ causes $s_{2}$ to malfunction (due to extraordinary crosstalk or delay variation) and vice-versa. In this case, $s_{1}$ is an aggressor for $s_{2}$, and $s_{2}$ a victim of $s_{1}$. The sensitivity rate of $s_{i}$ is defined as the ratio of the number of aggressors for $s_{i}$ to the total number of nets. A shield is a wire directly connected to $\mathrm{P} / \mathrm{G}$ wires. By inserting a shield between two sensitive s-wires, we are able to eliminate the capacitive coupling and reduce the inductive coupling.

In order to efficiently model inductive coupling between two arbitrary s-wires, the inductive coupling coefficient between two s-wires can be used as the figure of merit. The coupling coefficient between two nets $s_{i}$ and $s_{j}$ is defined as

$$
K_{i j}=\frac{m_{i j}}{\sqrt{l_{i} \cdot l_{j}}}
$$

where $m_{i j}$ is the mutual inductance between $s_{i}$ and $s_{j}$, and $l_{i}$ and $l_{j}$ is self inductance for $s_{i}$ and $s_{j}$, respectively. Let a block denote the set of wires between two shields. In this "effective coupling model" (i.e., $K_{\text {eff }}$ model), when the two nets are in the same block, as shown in Figure 1 where $N_{i}$ and $N_{j}$ are track ordering numbers for the two swires, and $g_{l}$ and $g_{r}$ are track ordering numbers for the two shields, the coupling coefficient is computed as

$$
K_{i, j}=\frac{(f(i)+g(j))}{2}
$$

where $f(i)$ and $g(j)$ are two functions defined as follows: assuming that the function $f$ is 0 at $g_{l}$, and is 1 at $N_{j}, f(i)$ is the linear interpolation, i.e., $f(i)=\frac{\left(N_{i}-g_{l}\right)}{\left(N_{j}-g_{l}\right)}$. Similarly, 
$g(j)=\frac{\left(g_{r}-N_{j}\right)}{\left(g_{r}-N_{i}\right)}$. When nets $i$ and $j$ are in different blocks, the coupling coefficient $K_{i}$ is 0 or a small constant. We compute the total inductive coupling for $s_{i}$ as:

$$
K_{i}=\sum_{j \neq i} K_{i j}
$$

for each s-wire $s_{j}$ that is sensitive to $s_{i}$.

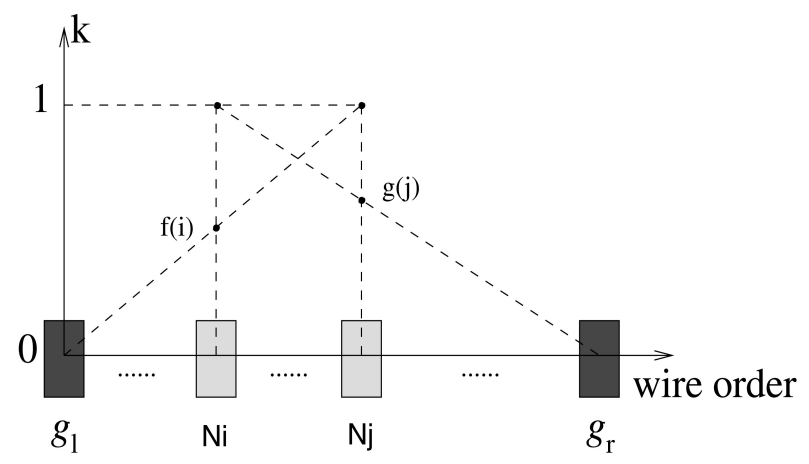

Figure 1. Illustration of $K_{e f f}$ computation.

The $K_{\text {eff }}$ model has been applied to SINO and SPR problems $[4,6]$. The SINO problem performs simultaneous shield insertion and net ordering. The SPR problem defines an optimal $\mathrm{P} / \mathrm{G}$ structure such that the minimal number of shields is needed by the optimal SINO solution with respect to the above $\mathrm{P} / \mathrm{G}$ structure. Both problems minimize the total routing area. Further, resulting solutions are free of capacitive coupling and satisfy inductive coupling constraint under the $K_{\text {eff }}$ model.

\section{Fideltiy of $K_{e f f}$ model}

The $K_{\text {eff }}$ model is easy to compute and convenient to use at a higher design level or at an earlier design stage. However, it is not clear how good the $K_{\text {eff }}$ model is as a figure of merit for the accurate noise voltage. Especially, it assumes that the current returns from the nearest shield, which may not be true in general. The current often returns from quiet wires within the current block if there are plenty of quiet wires in this block. On the other hand, the current often returns from shields or quiet wires outside the current block when multiple wires in the current block switch simultaneously. Despite that the $K_{\text {eff }}$ model is far away from accurate, it has a high fidelity in the following sense: an optimal SINO solution with a higher coupling value $K_{i}$ also has a higher SPICE-computed noise over a distributed RLC circuit model using the partial inductance model. Our observation is based on the following experiment. We first generate a large number of optimal SINO solutions using
SINO algorithms, and rank these solutions by both the coupling value $K_{i}$ and SPICE-computed noise using an accurate RLC circuit model. We then compute the absolute difference between the two rankings for each optimal SINO solution. If the ranking difference is small enough, the $K_{\text {eff }}$ model has a high fidelity. The similar ranking techniques have been used to study the fidelity of Elmore delay model in $[1,2]$.

Further, we calculate the SPICE-computed noise difference with respect to the average ranking difference in the following way: let the average ranking difference be $d$. For a SINO solution whose SPICE-computed noise ranking is $i$, we compute the relative difference between the $(i+d)$-th and $i$-th SPICE-computed noise, as well as that between the $(i-d)$-th and $i$-th SPICE-computed noise. Between the two values, the one with the larger absolute value divided by the $i$-th SPICE-computed noise value is defined as noise difference for the solution. In other words, it shows how much the noise difference is introduced if we use $K_{\text {eff }}$ model to approximate the SPICE-computed noise voltage under an accurate RLC circuit model.

\begin{tabular}{|l|l|}
\hline technology & $0.10 \mu \mathrm{m}$ \\
\hline \hline Vdd & $1.05 \mathrm{~V}$ \\
\hline frequency & $3 \mathrm{GHz}$ \\
input rising time & $33 \mathrm{ps}$ \\
\hline driver resistance & $150 \Omega$ \\
load capacitance & $60 \mathrm{fF}$ \\
\hline wire width & $1.0 \mu \mathrm{m}$ \\
wire thickness & $1.1 \mu \mathrm{m}$ \\
wire spacing & $0.8 \mu \mathrm{m}$ \\
wire length & $2000 \mu \mathrm{m}$ \\
\hline$K_{t h}$ & $0.5,1.0,1.5,2.0$ \\
\hline
\end{tabular}

\section{Table 1. Experiment settings for SPICE simu- lation}

In our SPICE simulation, we generate a RLC segment for each $100 \mu \mathrm{m}$ wire segment, and a mutual inductance between any pair of two segments even though they belong to the same wire. We use the partial inductance model in $[8,3]$, without assuming any current return path. The parameters for the SPICE simulation of the detailed RLC circuit model are summarized in Table 1. We report in Figure 2 the distribution of the ranking differences for SINO solutions. The average ranking difference is only 124.533 over 3,840 SINO solutions. The average noise difference with respect to such a ranking difference is $6.2018 \%$. In addition, we present in Table 2 the average noise difference for SINO solutions with different coupling values. One can easily see that the smaller the coupling, the smaller the average noise difference. We assume in our experiment that the largest 
coupling value is 2.0 under the $K_{\text {eff }}$ model. This value is chosen based on our experiments [4] that for $0.10 \mu \mathrm{m}$ technology, $K_{t h}=2.0$ is a "good" noise bound in the sense that the resulting SINO solutions have the SPICE-computed maximum noise close to but still less than $15 \%$ of $\mathrm{Vdd}$.

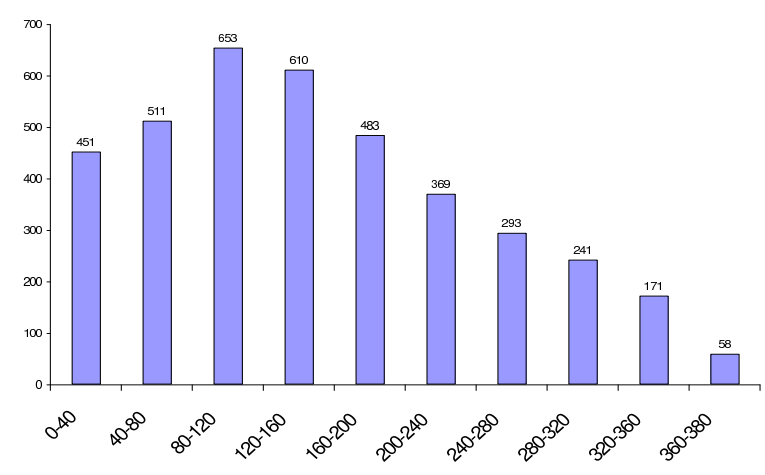

Figure 2. The distribution of the ranking difference for 3,840 SINO solutions.

\begin{tabular}{|c|c|}
\hline coupling value & noise difference \\
\hline $0<K_{i}<0.5$ & $5.1193 \%$ \\
$0.5<K_{i}<1.0$ & $5.9872 \%$ \\
$1.0<K_{i}<1.5$ & $6.5520 \%$ \\
$1.5<K_{i}<2.0$ & $7.1487 \%$ \\
\hline
\end{tabular}

Table 2. The average noise difference for different coupling values

Based on the above empirical evidence, we conclude that the $K_{e f f}$ model has a high fidelity over SPICE-computed noise using an accurate circuit model. This fidelity holds for optimal SINO solutions under practical noise bound. We speculate that the SINO algorithms are able to distribute shields and quite wires evenly both spatially and temporally, therefore the current mainly returns within the current block including the nearest shields. This leads to the fidelity of the $K_{\text {eff }}$ model.

\section{Formulae of pre-routing estimation}

We first consider the uniform sensitivity case, where each net has the same sensitivity rate $S$. Further, We denote the number of shields as $N_{s}$, the number of nets as $N$, and the coupling coefficient threshold as $K_{t h}$. As shown in [6], the number of shields for a fixed $K_{t h}$ is

$$
\begin{aligned}
N_{s}= & \left(a_{1} \cdot N+a_{2}\right) \cdot S^{2}+\left(a_{3} \cdot N+a_{4}\right) \cdot S \\
& +a_{5} \cdot N+a_{6}
\end{aligned}
$$

where $a_{1}$ to $a_{6}$ are coefficients determined empirically. However, the above formula did not consider $K_{t h}$ as a variable, which limits its application.

We propose that the number of shields is a function of $N, S$, and $K_{t h}$ as

$$
\begin{aligned}
N_{s}= & -0.448 \cdot K_{t h} \cdot N \cdot S^{2}+0.624 \cdot N \cdot S^{2} \\
& +21.67 \cdot K_{t h} \cdot S^{2}-29.87 \cdot S^{2} \\
& +0.384 \cdot K_{t h} \cdot N \cdot S-0.337 \cdot N \cdot S \\
& -22.83 \cdot K_{t h} \cdot S+31.45 \cdot S-0.12 \cdot K_{t h} \cdot N \\
& +0.202 \cdot N+5.47 \cdot K_{t h}-6.28
\end{aligned}
$$

This formula shows that given fixed values of $N$ and $S, N_{s}$ is a linear function of $K_{t h}$.

Formula (2) can be extended to the case with nonuniform sensitivity rates. When the sensitivity rate is not uniform but is $S_{i}$ for s-wire $s_{i}$, the number of shields is given by

$$
\begin{aligned}
N_{s}= & -0.448 \cdot K_{t h} \cdot \sum_{i=1}^{N} S_{i}^{2}+0.624 \cdot \sum_{i=1}^{N} S_{i}^{2} \\
& +21.67 \cdot \frac{K_{t h}}{N} \cdot \sum_{i=1}^{N} S_{i}^{2}-29.87 \frac{1}{N} \cdot \sum_{i=1}^{N} S_{i}^{2} \\
& +0.384 \cdot K_{t h} \cdot \sum_{i=1}^{N} S_{i}-0.337 \cdot \sum_{i=1}^{N} S_{i} \\
& -22.83 \cdot \frac{K_{t h}}{N} \cdot \sum_{i=1}^{N} S_{i}+31.45 \frac{1}{N} \cdot \sum_{i=1}^{N} S_{i} \\
& -0.12 \cdot K_{t h} \cdot N+0.202 \cdot N+5.47 \cdot K_{t h} \\
& -6.28
\end{aligned}
$$

We verify formulae (2) and (3) by comparing numbers of shields given by the formulae and those obtained by the SINO program. As shown in Figure 3, the difference between the estimation and the SINO program is less than $10 \%$. Note that we can tune the constant in formulae (2) and (3) to obtain a desired lower or upper bound on the number of shields required by SINO solutions.

\section{Conclusion and discussion}

In this paper, we have shown that the $K_{\text {eff }}$ model has a high fidelity to SPICE-computed noise under an accurate RLC circuit model. We have developed simple yet accurate formulae to estimate the number of shields needed by optimal SINO solutions under the $K_{\text {eff }}$ model. Extensive experiments show that our pre-routing estimation has errors less than $10 \%$ compared to solutions given by detailed SINO algorithms. Such estimation can be used by interconnect planning and synthesis. For example, shielding estimation has been used to develop an efficient two-phase algorithm for the SPR problem [6]. Further, we have recently 


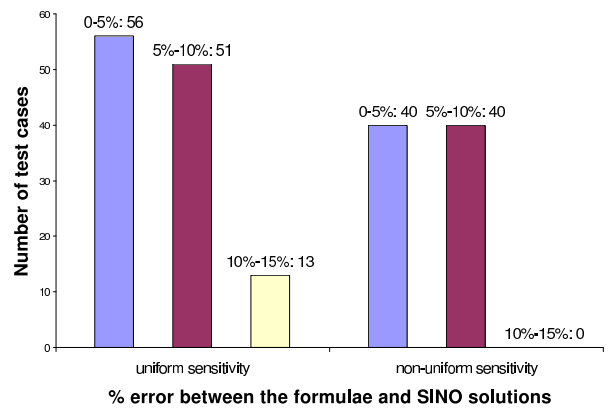

\section{Figure 3. Comparison of $N_{s}$ by the estimation} and the SINO- $k$ program.

developed shielding estimation for SINO solutions under an explicit RLC noise constraint [5, 7].

The simplest interconnect structure, parallel bus structure, is assumed in this work. We are incorporating the prerouting estimation and SPR formulation to a global router for signal and power net co-design. Even though the explicit RLC noise model used in $[5,7]$ is more accurate, we plan to first apply the $K_{\text {eff }}$ model to the global router due to the simplicity and high fidelity of the $K_{\text {eff }}$ model. These new results will be made available at http://eda.ece.wisc.edu.

\section{References}

[1] K. D. Boese, A. B. Kahng, and G. Robins. High-performance routing trees with identified critical sinks. In Proc. Design Automation Conf, pages 182-187, 1993.

[2] J. Cong and L. He. Optimal wiresizing for interconnects with multiple sources. ACM Trans. on Design Automation of Electronics Systems, 1996.

[3] L. He, N. Chang, S. Lin, and O. S. Nakagawa. An efficient inductance modeling for on-chip interconnects. In Proc. IEEE Custom Integrated Circuits Conference, pages 457-460, May 1999.

[4] L. He and K. M. Lepak. Simultaneous shield insertion and net ordering for capacitive and inductive coupling minimization. In Proc. Int. Symp. on Physical Design, 2000.

[5] K. M. Lepak, I. Luwandi, and L. He. Simultaneous shield insertion and net ordering for coupled rlc nets under explicit noise constraint. In Proc. Design Automation Conf, pages 199-202, 2001.

[6] J. D. Ma and L. He. Simulatenous signal and power routing under $k_{\text {eff }}$ model. The 3rd Intl. Workshop on System-Level Interconnect Prediction, 2001.

[7] J. D. Z. Ma and L. He. Formulae and applications of interconnect estimation considering shield insertion and net ordering. Accepted and to appear in International Conference on Computer-Aided Design, 2001.

[8] A. E. Ruehli. Equivalent circuit models for three-dimensional multiconductor systems. IEEE Trans. on MTT, 1974. 